\title{
Communication
}

\section{Surfactant-Template Preparation of Polyaniline Semi-Tubes for Oxygen Reduction}

\section{Shiming Zhang and Shengli Chen *}

Hubei Key Laboratory of Electrochemical Power Sources, Department of Chemistry, Wuhan University, Wuhan 430072, China; E-Mail: zsm19860409@163.com

* Author to whom correspondence should be addressed; E-Mail: slchen@whu.edu.cn; Tel.: +86-027-68754693; Fax: +86-027-68754693.

Academic Editor: Minhua Shao

Received: 13 May 2015 / Accepted: 1 July 2015 / Published: 7 July 2015

\begin{abstract}
Nitrogen and metal doped nanocarbons derived from polyaniline (PANI) have been widely explored as electrocatalysts for the oxygen reduction reaction (ORR) in fuel cells. In this work, we report surfactant-template synthesis of PANI nanostructures and the ORR electrocatalysts derived from them. By using cationic surfactant such as the cetyl trimethyl ammonium bromide (CTAB) as the template and the negatively charged persulfate ions as the oxidative agent to stimulate the aniline polymerization in the micelles ofCTAB, PANI with a unique 1-D semi-tubular structure can be obtained. The semi-tubular structure can be maintained even after high-temperature treatment at $900{ }^{\circ} \mathrm{C}$, which yields materials exhibiting promising ORR activity.
\end{abstract}

Keywords: polyaniline; cetyl trimethyl ammonium bromide; micelle; semi-tubes; oxygen reduction reaction

\section{Introduction}

Seeking the highly-active electrocatalysts for oxygen reduction reaction (ORR) has become the one of the urgent demands for fuel cells, which would take a key role in the "hydrogen energy economy" [1]. In recent years, non-precious metal and/or metal-free materials based on nitrogen (N)-doped nanocarbons have shown great promise in substituting Pt and its alloys for catalyzing the ORR $[2,3]$. 
Polyaniline (PANI), a low-cost and easy-making conjugate conducting polymer containing rich content of nitrogen, has received extensive research interest [4-6]. Very recently, a variety of N-doped carbon catalysts based on PANI have been constructed which showed superior electrocatalytic activities for the ORR [7-12]. The multi-technique characterization have suggested that the enviable performance should be ascribed to the formation of metal-N complexion structures as well as the carbon nanostructures such as thin graphene sheets and nanofibers [10-12]. It has been generally accepted that the formation of uniform and ordered carbon nanostructures is very important in enhancing the catalytic activity [10-19].

Up to now, doped carbon electrocatalysts of different morphologies, such as nanoparticles [20], nanowires [21], nanotubes [22], nanorods [23], hollow nanospheres [24] and amorphous carbons [25], have been constructed by using various methods. The soft-template synthesis through self-assembly processes is among the most straightforward methods for nanostructure formation. In this work, we use assembly architectures of a variety of surfactants as the soft-templates to synthesize PANI nanostructures. In particular, PANI semi-tubes with uniform diameters of $\sim 80 \mathrm{~nm}$ are obtained by using cationic surfactant. The electrocatalysts derived from these PANI semi-tubes show good ORR catalytic activity in alkaline.

\section{Results and Discussion}

Figure 1 shows the morphologies of PANI materials obtained by using CTAB $(80 \mathrm{mM})$ as the template and APS as the oxidative agent. It can be seen that uniform 1-D nanostructures with diameters of $\sim 80 \mathrm{~nm}$ and lengths of a few micrometers were obtained under this condition. Careful inspection revealed that these 1-D nanostructures possessed semi-cannular structures. As seen from the TEM images (Figure $1 \mathrm{~b}$ and its insert), the walls of the individual tubes were highly rugged and full of cone-shaped protuberances of $\sim 10 \mathrm{~nm}$ lengths, exhibiting centipede-like morphologies. We denoted this sample as $\mathrm{PANI}_{s-\text { tubes. }}$

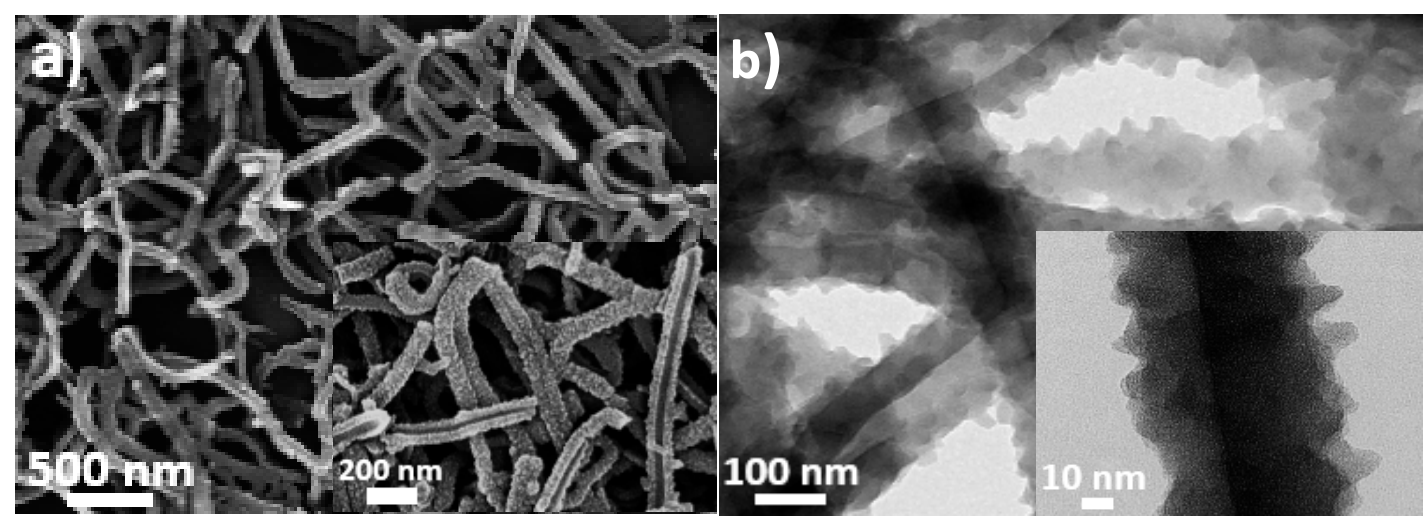

Figure 1. (a) SEM and (b) TEM images for polyaniline (PANI) obtained by using cetyl trimethyl ammonium bromide (CTAB) as template and ammonium persulfate (APS) as oxidative agent.

In the case when the preparation was conducted using the same procedure as that giving $\mathrm{PANI}_{s-t u b e s}$ but the CTAB was absent, highly agglomerated PANI particles were obtained (Figure 2a). When the $\mathrm{FeCl}_{3}$ was used to replace APS as the oxidative agent to stimulate the polymerization of aniline in 
CTAB solution, irregular PANI nanosheets were obtained (Figure 2b). These results indicated that the semi-cannular structured PANIs can be uniquely formed through the oxidative polymerization of anilines by APS in the assemblies of CTAB.

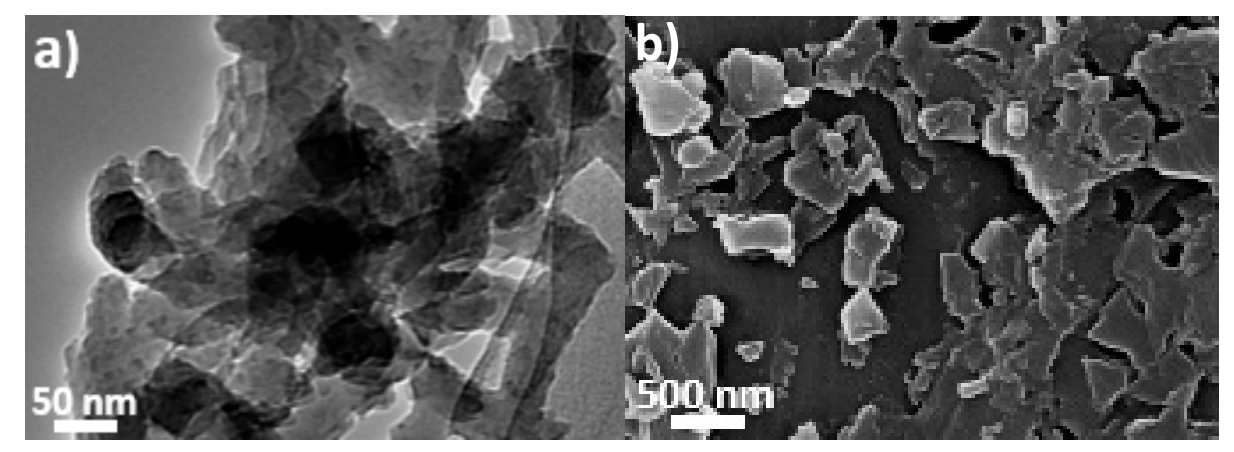

Figure 2. Morphologies of PANI materials obtained when (a) the CTAB was absent, or (b) $\mathrm{FeCl}_{3}$ instead of APS was used as the oxidative reagent. The other conditions are the same as that for Figure 1.

We have also explored the effects of the surfactant types on the morphologies of the formed PANI materials. For anionic surfactants, e.g., SDBS, and non-ionic surfactants, e.g., X-100 and Span 40, mixtures of PANI nanoparticles and nanorods were obtained (Figure 3), which indicated the uniqueness of CTAB in producing the tubular structures of PANI.

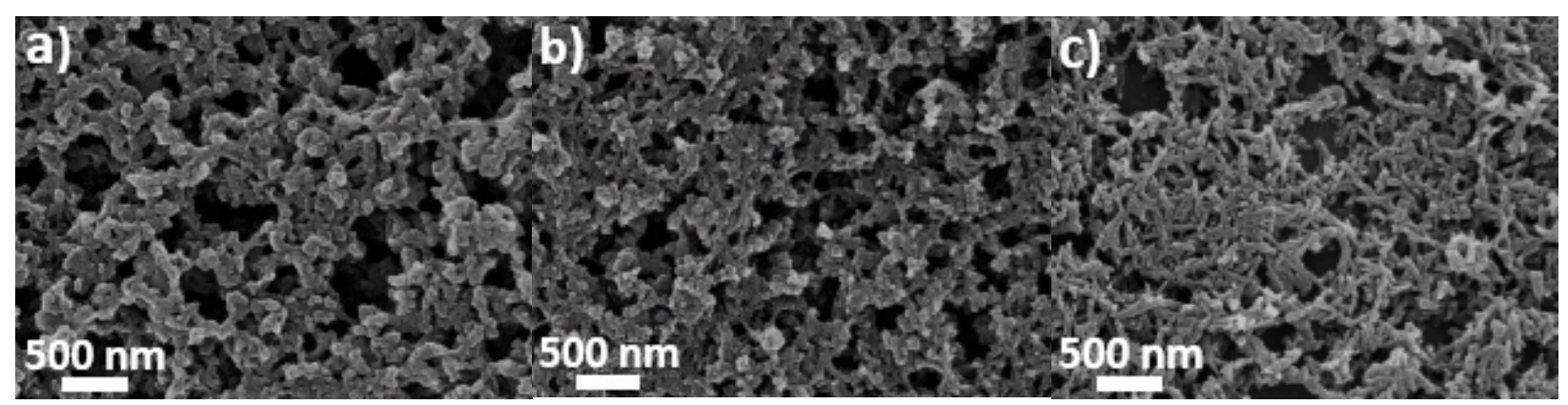

Figure 3. Morphologies of PANI materials obtained when the CTAB was replaced by (a) sodium dodecyl benzene sulfonate (SDBS), (b) Triton X-100 or, (c) Span 40. The other conditions are the same as that for Figure 1.

We believed that the formation of the PANI semi-tubes was related to the rod-like CTAB micelles and the opposite charges between the oxidative persulfate ions and the protonated aniline. Scheme 1 depicts the possible growth mechanism. The aniline molecules should be dissolved into the rod-like CTAB micelles. In the presence of $\mathrm{HCl}$, the aniline molecules should be protonated. Therefore, they would be mainly located in the outer region of the micelles. The electrostatic attraction made the negatively charged persulfate ions approach the outer surface of micelles and oxidize the aniline molecules, which stimulate the polymerization in the outer region of cylindrical micelles. The formation of half instead of full PANI tubes was probably due to that there were only limited amounts of aniline molecules dissolved in the micelles. When the CTAB micelles were present or positively charged Fe(III) ions were used as oxidative agent, the mechanism shown in Scheme 1 could be altered. A deeper 
understand ing of the effects of the oxidants and surfactants on the resultant PANI morphologies requires much more detailed investigation.

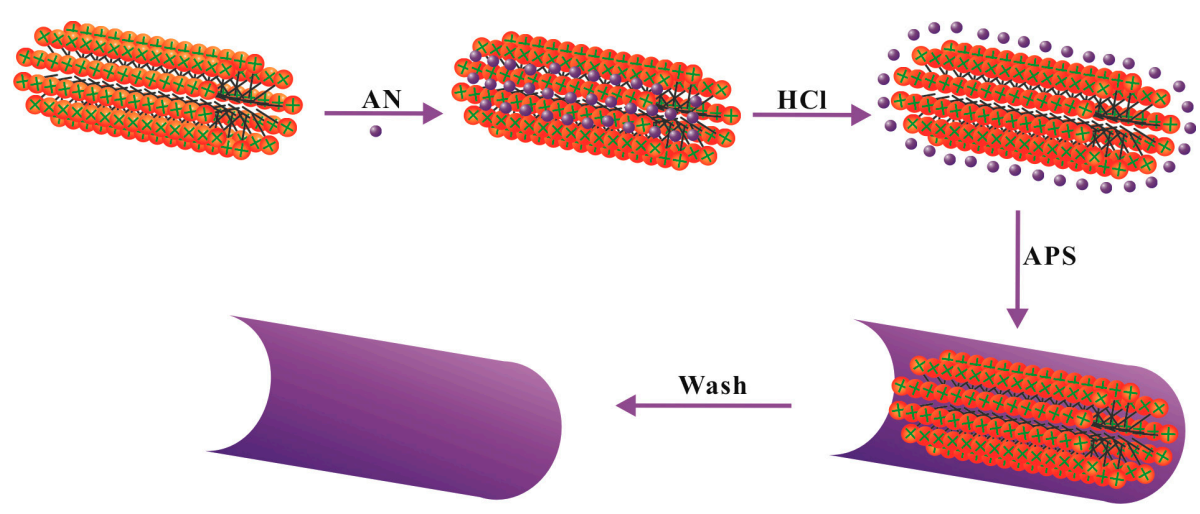

Scheme 1. The possible growth process of 1-D PANIs-tubes by CTAB micelle as the soft-template.

The obtained PANI samples were converted into N-doped carbon materials by heat-treating at $900{ }^{\circ} \mathrm{C}$ under Ar atmosphere. As shown in Figure 4, semi-tubular morphologies were maintained after the heat-treatment.

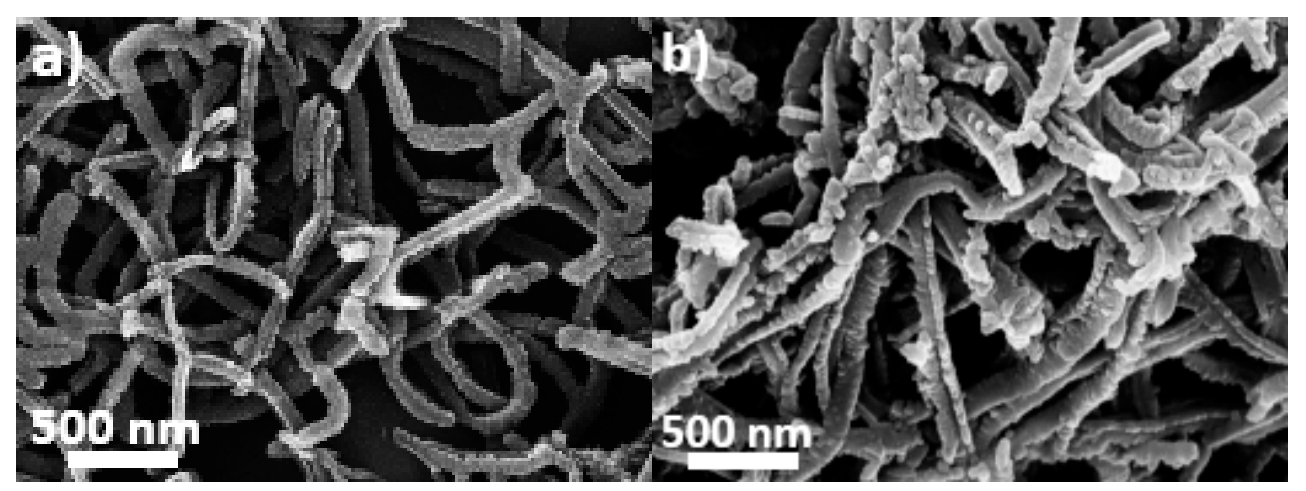

Figure 4. Comparison between the morphologies of $\mathrm{PANI}_{s \text {-tubes }}$ (a) before and (b) after heat-treatment at $900^{\circ} \mathrm{C}$.

We investigated the physical properties and chemical composition of the $\mathrm{PANI}_{s \text {-tubes }}$ materials before and after the heat-treatment. It was found that the heat-treatment resulted in materials exhibiting BET surface area $\left(c a .351 .8 \mathrm{~m}^{2} / \mathrm{g}\right)$ and pore volume $\left(0.45 \mathrm{~m}^{3} / \mathrm{g}\right)$ which were much higher than that before heat-treatment ( $c a .54 .8 \mathrm{~m}^{2} / \mathrm{g}$ and $0.29 \mathrm{~m}^{3} / \mathrm{g}$ respectively). This was probably due to the volatilizing release of some components during the heat-treatment, making the resulted materials more porous. XPS characterization results indicated that the contents of $\mathrm{N}$ and $\mathrm{O}$ decreased significantly after heat-treatment (Table 1), which confirmed the volatilization of some components. 
Table 1. The content values of $\mathrm{C}, \mathrm{N}$ and $\mathrm{O}$ estimated from XPS results for $\mathrm{PANI}_{s-\text { tubes }}$ before and after heat-treatment.

\begin{tabular}{cccc}
\hline PANI $_{\text {stubes }}$ & C (at. \%) & N (at. \%) & O (at. \%) \\
\hline After Heat-Treatment & 88.96 & 5.79 & 5.25 \\
Before Heat-Treatment & 78.85 & 10.88 & 10.27 \\
\hline
\end{tabular}

Figure 5 compares the ORR polarization curves of the materials obtained through heat-treating the PANI prepared without using surfactant (see Figure 2a), the PANI stubes $_{\text {prepared by using CTAB }}$ micelles as templates, and the $\mathrm{PANI}_{s \text {-tubes }} / \mathrm{GS}$ composite. For comparison, the ORR polarization curve for the pure GS is also given. It can be seen that the heat-treated $\mathrm{PANI}_{s-\text { tubes }}$ exhibited significantly enhanced ORR activity as compared with the material derived from the PANI that was prepared without using surfactant template. This should be due to the open semi-tubular structure, which gave higher specific surface areas. As compared with the heat-treated PANI positive ORR onset potential, but slower current rising rate and lower limiting current. The heat-treated $\mathrm{PANI}_{s \text {-tubes }} / \mathrm{GS}$ composite showed much higher ORR activity than that exhibited by the heat-treated $\mathrm{PANI}_{s \text {-tubes }}$ and the GS alone.

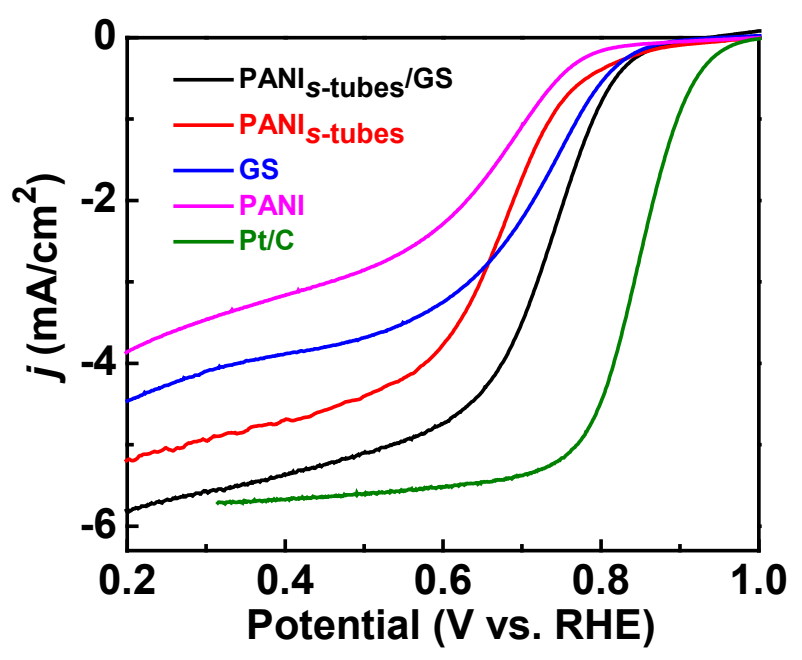

Figure 5. ORR polarization curves for different catalyst samples in $\mathrm{O}_{2}$-saturated $0.1 \mathrm{M} \mathrm{KOH}$ at an electrode rotation speed of $1600 \mathrm{rpm}$. The PANI refers to the sample obtained by heat-treatment of PANI prepared without using surfactant; the $\mathrm{PANI}_{s \text {-tubes }} / \mathrm{GS}$ refers to the sample obtained by heat-treatment of $\mathrm{PANI}_{s \text {-tubes }}$ and graphene sheets together. The catalyst load ings were $0.3 \mathrm{mg} \mathrm{cm}^{-2}$ for the non-precious metal catalysts and $0.1 \mathrm{mg} \mathrm{cm}^{-2}$ for $\mathrm{Pt} / \mathrm{C}\left(20 \mu \mathrm{g} \mathrm{cm}^{-2}\right.$ for $\left.\mathrm{Pt}\right)$.

Since the ORR polarization curves in Figure 5 were obtained with same total mass loading of $0.3 \mathrm{mg} \mathrm{cm}^{-2}$ for the heat-treated PANI $\mathrm{P}_{\text {-tubes, }}, \mathrm{GS}$ and $\mathrm{PANI}_{s \text {-tubes }} / \mathrm{GS}$, one may expect that the ORR activity of the heat-treated $\mathrm{PANI}_{s \text {-tubes }} / \mathrm{GS}$ composite is between that of the heat-treated $\mathrm{PANI}_{s-\text { tubes }}$ and GS. The actually higher ORR activity of the composite thus indicated that there were synergetic interaction between $\mathrm{PANI}_{s-\text { tubes }}$ and GS. The introduction of GS may increase the electrical conductivity of the composite. On the other hand, the formation of composite between the 2-D GS and the 1-D $\mathrm{PANI}_{s \text {-tubes }}$ would prevent the GS and $\mathrm{PANI}_{\text {s-tubes }}$ from agglomerating and stacking during 
the heat treatment and electrode preparation. There have been numerous studies showing that the electrochemical performance of nanomaterials can be enhanced by forming composites with GS, due to the good electric conductivity of GS and the capability of GS to improve the dispersion of the electroactive materials [26,27].

It can be seen that the limiting current of the composite was very similar to that of the $\mathrm{Pt} / \mathrm{C}$ electrocatalyst. The value of the limited current is directly related to the electron transfer number of the reaction. It is known that the Pt-based electrocatalysts catalyze the ORR through a 4-electron pathway. Therefore, we have reason to believe that ORR proceeded on the heat-treated composite mainly through a 4-electron process. It is noted that the present $\mathrm{PANI}_{s-\text { tubes }}$ materials were still less efficient for the ORR than the $\mathrm{Pt} / \mathrm{C}$ catalyst. Further optimization on the material preparation is necessary to promote the electrocatalytic activity.

\section{Experimental Section}

\subsection{Chemicals and Materials}

Various surfactants, such as cetyl trimethyl ammonium bromide (CTAB), sodium dodecyl benzene sulfonate (SDBS), and octoxinol (Triton X-10), and sorbitan monopalmitate (Span 40), and other chemicals were purchased from Sinopharm Chemical Reagent Co., Ltd. (Shanghai, China). The 20 wt. \% Pt/C from Johnson Matthey (JM, London, UK) was used as reference catalyst.

\subsection{Materials Synthesis}

In a typical synthesis, a desired amount of CTAB was mixed with $15 \mathrm{~mL}$ of ultrapure water under ultrasonication for more than $30 \mathrm{~min}$. After fully dissolution of CTAB, $200 \mu \mathrm{L}$ of aniline (AN) and $20 \mathrm{~mL}$ of $1 \mathrm{M} \mathrm{HCl}$ were successively added under ultrasonication for another $30 \mathrm{~min}$ and then the solution was allow to stay for $24 \mathrm{~h}$. $5 \mathrm{~mL}$ of $0.4 \mathrm{M}$ ammonium persulfate (APS) solution was then added into the solution to stimulate the polymerization of aniline in the CTAB assemblies. Different CTAB concentrations (10-80 $\mathrm{mM}$ ) were explored and no substantial difference in the morphology was seen for the obtained PANI. During the reaction progress, the color gradually changed to blue and cloudy precipitates were formed, which were collected through ultrafiltration and then alternately washed by ethanol and water followed by freeze-drying. Finally, dark blue product was obtained. For comparison, the preparation was also conducted by replacing CTAB with other surfactants.

We also prepared the composite of PANIs with graphene nanosheets (GS) that were prepared by high temperature thermal reduction of graphene oxide [17,18]. In this case, $30 \mathrm{mg}$ of GS were added into the CTAB solution to prepare the PANI, which corresponded to a PANI/GS ratio of 1/8. Different PANI/GS ratios were explored and the $1 / 8$ was found to be the most optimized value. To obtain electrocatalysts from the obtained nanostructured PANI, they were pyrolyzed under a flow of Ar at $900{ }^{\circ} \mathrm{C}$ for $1 \mathrm{~h}$.

\subsection{Characterization}

Scanning electron microscopy (SEM) images were obtained by Hitachi S-4800 Scanning Electron Microsope (Tokyo, Japan). Transmission electron microscope (TEM) images were obtained at 
JEM-2100F (JEOL Ltd., Tokyo, Japan). The values of Brunner-Emmet-Teller (BET) surface area and total pore volume (TPV) were from $\mathrm{N}_{2}$ adsorption isotherms using an ASAP2020 Surface Area and Porosity Analyzer (Micromeritics, Atlanta, GA, USA). X-ray photoelectron spectroscopy (XPS) measurements were carried out using a Kratos Ltd. XSAM-800 spectrometer (Kratos Analytical Ltd., Manchester, UK) with $\mathrm{Mg} \mathrm{K} \alpha$ radiator. The data were fitted by using Gaussian/Lorentzian fitting in the software XPSPEAK41 (Kratos Analytical Ltd., Manchester, UK) with Shirley function as baseline.

\subsection{Electrochemical Measurement}

The three-electrode configuration were used for electrochemical measurements using Pt foil counter electrode and saturated calomel reference electrode. To prepare the working electrodes, catalyst samples as a thin film were coated onto a glass carbon (GC) RDE substrate (diameter: $5 \mathrm{~mm}$ ) with Nafion as the binding agent. For the PANI nanostructures, $5 \mathrm{mg}$ catalysts were dispersed in $1 \mathrm{~mL}$ Nafion solution ( 0.5 wt. \% Nafion in isopropyl alcohol) to form the catalyst inks and $12 \mu \mathrm{L}$ ink suspension was pipetted onto the GC RDE. For the Pt/C catalyst, $5 \mathrm{mg}$ catalyst was dispersed ultrasonically in $1 \mathrm{~mL}$ Nafion-isopropyl alcohol solution and $4 \mu \mathrm{L}$ of the resulted suspension was then pipetted onto the GC RDE. The catalyst loadings were respectively $0.3 \mathrm{mg} \mathrm{cm}^{-2}$ for the non-precious metal catalysts and $0.1 \mathrm{mg} \mathrm{cm}^{-2}$ for the $\mathrm{Pt} / \mathrm{C}\left(20 \mu \mathrm{g} \mathrm{cm}^{-2}\right.$ for $\left.\mathrm{Pt}\right)$.

\section{Conclusions}

In this work, a unique 1-D semi-tubular structure of PANI has been obtained by using self-assemblies of CTAB molecules as soft-templates and APS as oxidative agent in aqueous solution. The obtained PANI nanostructure can be maintained in the course of high-temperature treatment. The materials derived from heat-treating the composite of PANI semi-tubes and GS show significantly enhanced ORR performance.

\section{Acknowledgments}

This work was supported by the Ministry of Science and Technology of China under the National Basic Research Program (Grant nos. 2012CB215500 and 2012CB932800).

\section{Author Contributions}

S.M.Z. performed the experiments. S.L.C. and S.M.Z. analyzed the data and wrote the paper.

\section{Conflicts of Interest}

The authors declare no conflict of interest.

\section{References}

1. Handbook of Fuel Cells: Fundamentals, Technology and Application; Vielstich, W., Lamm, A., Gasteiger, H.A., Eds.; Wiley: West Sussex, UK, 2003.

2. Mazumder, V.; Lee, Y.; Sun, S. Recent Development of Active Nanoparticle Catalysts for Fuel Cell Reactions. Adv. Funct. Mater. 2010, 20, 1224-1231. 
3. Chen, Z.; Higgins, D.; Yu, A.; Zhang, L.; Zhang, J. A review on non-precious metal electrocatalysts for PEM fuel cells. Energy Environ. Sci. 2011, 4, 3167-3192.

4. Zhang, D.; Wang, Y. Synthesis and applications of one-dimensional nano-structured polyaniline: An overview. Mater. Sci. Eng. B 2006, 134, 9-19.

5. Li, D.; Huang, J.; Kaner, R.B. Polyaniline Nanofibers: A Unique Polymer Nanostructure for Versatile Applications. Acc. Chem. Res. 2008, 42, 135-145.

6. Wang, L.; Lu, X.; Lei, S.; Song, Y. Graphene-Based Polyaniline Nanocomposites: Preparation, Properties and Applications. J. Mater. Chem. A 2014, 2, 4491-4509.

7. Zhong, H.; Zhang, H.; Xu, Z.; Tang, Y.; Mao, J. A Nitrogen-Doped Polyaniline Carbon with High Electrocatalytic Activity and Stability for the Oxygen Reduction Reaction in Fuel Cells. ChemSusChem 2012, 5, 1698-1702.

8. Hu, Y.; Zhao, X.; Huang, Y.; Li, Q.; Bjerrum, N.J.; Liu, C.; Xing, W. Synthesis of Self-Supported Non-Precious Metal Catalysts for Oxygen Reduction Reaction with Preserved Nanostructures from the Polyaniline Nanofiber Precursor. J. Power Sources 2013, 225, 129-136.

9. Gavrilov, N.; Pašti, I.A.; Mitrić, M.; Travas-Sejdić, J.; Ćirić-Marjanović, G.; Mentus, S.V. Electrocatalysis of Oxygen Reduction Reaction on Polyaniline-Derived Nitrogen-Doped Carbon Nanoparticle Surfaces in Alkaline Media. J. Power Sources 2012, 220, 306-316.

10. Wu, G.; More, K.L.; Johnston, C.M.; Zelenay, P. High-Performance Electrocatalysts for Oxygen Reduction Derived from Polyaniline, Iron, and Cobalt. Science 2011, 332, 443-447.

11. Ferrandon, M.; Kropf, A.J.; Myers, D.J.; Artyushkova, K.; Kramm, U.; Bogdanoff, P.; Wu, G.; Johnston, C.M.; Zelenay, P. Multitechnique Characterization of a Polyaniline-Iron-Carbon Oxygen Reduction Catalyst. J. Phys. Chem. C 2012, 116, 16001-16013.

12. Wu, G.; Zelenay, P. Nanostructured Nonprecious Metal Catalysts for Oxygen Reduction Reaction. Acc. Chem. Res. 2013, 46, 1878-1889.

13. Gong, K.; Du, F.; Xia, Z.; Durstock, M.; Dai, L. Nitrogen-doped carbon nanotube arrays with high electrocatalytic activity for oxygen reduction. Science 2009, 323, 760-764.

14. Cheon, J.Y.; Kim, T.; Choi, Y.; Jeong, H.Y.; Kim, M.G.; Sa, Y.J.; Kim, J.; Lee, Z.; Yang, T.H.; Kwon, K.; et al. Ordered Mesoporous Porphyrinic Carbons with Very High Electrocatalytic Activity for the Oxygen Reduction Reaction. Sci. Rep. 2013, 3, 2715.

15. Ding, W.; Li, L.; Xiong, K.; Wang, Y.; Li, W.; Nie, Y.; Chen, S.; Qi, X.; Wei, Z. Shape Fixing via Salt Recrystallization: A Morphology-Controlled Approach To Convert Nanostructured Polymer to Carbon Nanomaterial as a Highly Active Catalyst for Oxygen Reduction Reaction. J. Am. Chem. Soc. 2015, 137, 5414-5420.

16. Zhang, S.; Zhang, H.; Hua, X.; Chen, S. Tailoring Molecular Architectures of Fe Phthalocyanine on Nanocarbon Supports for High Oxygen Reduction Performance. J. Mater. Chem. A 2015, 3, $10013-10019$.

17. Zhang, S.; Zhang, H.; Liu, Q.; Chen, S. Fe-N doped carbon nanotube/graphene composite: Facile synthesis and superior electrocatalytic activity. J. Mater. Chem. A 2013, 1, 3302-3308.

18. Zhang, S.; Liu, B.; Chen. S. Synergistic increase of oxygen reduction favourable Fe-N coordination structures in a ternary hybrid of carbon nanospheres/carbon nanotubes/graphene sheets. Phys. Chem. Chem. Phys. 2013, 15, 18482-18490. 
19. Liu, Q.; Zhang, H.; Zhong, H.; Zhang, S.; Chen. S. N-doped graphene/carbon composite as non-precious metal electrocatalyst for oxygen reduction reaction. Electrochim. Acta 2012, 81, 313-320.

20. Yan, J.; Wei, T.; Fan, Z.; Qian, W.; Zhang, M.; Shen, X.; Wei, F. Preparation of Graphene Nanosheet/Carbon Nanotube/Polyaniline Composite as Electrode Material for Supercapacitors. J. Power Sources 2010, 195, 3041-3045.

21. Xu, J.; Wang, K.; Zu, S.Z.; Han, B.H.; Wei, Z. Hierarchical Nanocomposites of Polyaniline Nanowire Arrays on Graphene Oxide Sheets with Synergistic Effect for Energy Storage. ACS Nano 2010, 4, 5019-5026.

22. Huang, Y.; Lin, C. Facile Synthesis and Morphology Control of Graphene Oxide/Polyaniline Nanocomposites via in situ Polymerization Process. Polymer 2012, 53, 2574-2582.

23. Hu, L.; Tu, J.; Jiao, S.; Hou, J.; Zhu, H.; Fray, D.J. In situ Electrochemical Polymerization of a Nanorod-PANI-Graphene Composite in a Reverse Micelle Electrolyte and Its Application in a Supercapacitor. Phys. Chem. Chem. Phys. 2012, 14, 15652-15656.

24. Fan, W.; Zhang, C.; Tjiu, W.W.; Pramoda, K.P.; He, C.; Liu, T. Graphene-Wrapped Polyaniline Hollow Spheres as Novel Hybrid Electrode Materials for Supercapacitor Applications. ACS Appl. Mater. Interfaces 2013, 5, 3382-3391.

25. Lai, L.; Potts, J.R.; Zhan, D.; Wang, L.; Poh, C.K.; Tang, C.; Gong, H.; Shen, Z.; Linc, J.; Ruoff, R.S. Exploration of the Active Center Structure of Nitrogen-Doped Graphene-Based Catalysts for Oxygen Reduction Reaction. Energy Environ. Sci. 2012, 5, 7936-7942.

26. Lee, S.H.; Lee, D.H.; Lee, W.J.; Kim, S.O. Tailored Assembly ofCarbon Nanotubes and Graphene. Adv. Funct. Mater. 2011, 21, 1338-1354.

27. Fan, Z.; Yan, J.; Zhi, L.; Zhang, Q.; Wei, T.; Feng, J.; Zhang, M.; Qian, W.; Wei, F. A Three-Dimensional Carbon Nanotube/Graphene Sandwich and Its Application as Electrode in Supercapacitors. Adv. Mater. 2010, 22, 3723.

(C) 2015 by the authors; licensee MDPI, Basel, Switzerland. This article is an open access article distributed under the terms and conditions of the Creative Commons Attribution license (http://creativecommons.org/licenses/by/4.0/). 